\title{
Screening Foliage Plants for Indoor Air Pollution Abatement
}

\author{
Randani M.M.N. ${ }^{1 *}$, Caldera H.I.U. ${ }^{1}$, Kaumal M.N. ${ }^{2}$ \\ ${ }^{1}$ Department of Plant Science, University of Colombo, Sri Lanka \\ ${ }^{2}$ Department of Chemistry, University of Colombo, Sri Lanka \\ *naleekarandani@gmail.com
}

\begin{abstract}
Indoor air pollution is a major environmental health problem affecting people in both developed and developing countries. The use of houseplants is one of the emerging methods for abatement of indoor air pollution. Plants can remove indoor air pollutants by stomatal uptake, absorption and adsorption to plant surfaces. Indoor air characteristically contains a large consortium of volatile organic compounds (VOCs), such as formaldehyde, benzene and toluene. The volatile tested in this study toluene, is commonly found in buildings. The main objective of this project was to find the removal efficiency of toluene by four plant species, Aglaonema spp., Dracaena deremensis, Hedera helix and Chlorophytum comosum. This main goal was achieved by constructing a sealed closed chamber resembling the indoor environment in order to find the activity of selected indoor plants to the indoor air pollutant toluene. The concentration of introduced toluene was measured through gas-chromatography mass spectrometry (GC/MS). The possible causes for the differences in removal of toluene among selected plant species were determined by studying leaf morphology, stomatal numbers, plant physiology and growth related parameters. The removal efficiency, expressed on percentage level using toluene peak areas of the GCMS analyses varied among plant species. H. helix had the highest toluene removal percentage per plant over 24 hours $(91.1 \%)$ followed by $D$. deremensis $(75.0 \%), A$. spp. (60.5\%) and C. comosum (47.9\%). The study found that stomatal index and stomatal pore length play a key role in VOC removal and that there was a positive relationship between stomatal conductance with toluene removal potential. Based on the results it can be further concluded that thickness of the cuticle is a hindrance to VOC penetration. In addition VOC removal is higher in plants having high photosynthetic rates and there was no relationship of leaf area with the VOC removal capacity. Further studies focusing on screening additional plant species for superior VOC removal efficiencies are warranted.
\end{abstract}

Keywords: Indoor air pollution, Volatile organic compounds, Gas-chromatography Mass spectrometry, Stomatal conductance 